\title{
Hospital Pharmacists' Documentation of Vancomycin and Aminoglycoside Prescriptive Authority
}

\author{
Brandon Kennedy, Carolyn Gray, Lisa Ruda, and William Semchuk
}

Can J Hosp Pharm. 2021;74(2):144-8

\section{INTRODUCTION}

As pharmacists advance in direct patient care roles, it is important that their clinical decisions be recorded in the medical record to ensure continuity of care. Multiple pharmacy professional organizations have emphasized documentation as a critical component of the provision of collaborative patient care by health care professionals. ${ }^{1-3}$ The expanding scope of practice for pharmacists in Saskatchewan includes collaborative prescriptive authority, which requires an explicit prescribing agreement between physicians and pharmacists, based on shared responsibilities and liabilities. ${ }^{4-6}$ The Saskatchewan College of Pharmacy Professionals (SCPP) defines documentation as a critical component of the collaborative practice agreement. ${ }^{4-6}$

In the Saskatchewan Health Authority (SHA) - Regina area, the Regina Pharmacy and Therapeutics Committee has authorized a practice agreement enabling pharmacists to serve as collaborative prescribers of vancomycin and aminoglycosides for adult patients receiving acute care. The agreement stipulates that the pharmacist will initiate and maintain documentation as per current Pharmacy Department procedures for all patients who are receiving vancomycin and aminoglycosides. Furthermore, the agreement specifies that the pharmacist will document all interventions and recommendations regarding dose determination, serum drug levels, phone calls with prescribers, nephrotoxicity risk factors, need for increased monitoring, and other pertinent information in the chart, whether or not the regimen is changed.

A Steering Committee consisting of pharmacists and infectious disease physicians developed a procedure for pharmacists to use in operationalizing the agreement, which outlines in detail dose determination, monitoring, and documentation expectations. All pharmacists in our organization undergo a certification process before participating in the agreement. Certification includes a review of protocols and procedures, case-based application, and a written examination incorporating a documentation component.
Previous local research identified gaps in the frequency of documentation and omission of specific components of the progress note. ${ }^{7,8}$ Tangedal and others ${ }^{8}$ assessed prescriptive authority competency related to application of the vancomycin protocol. They identified deficiencies in documentation relating to aspects such as nephrotoxic risk factors, requirements for future trough levels, and validity of trough samples drawn (i.e., relative to start time and at steady state).

A pharmacists' working group (the Documentation Working Group or DWG) was established in November 2016 to facilitate increased documentation of all pharmacists' interventions in the progress notes of the patient chart. ${ }^{9}$ As one part of their targeted activities, the group sought to determine the extent of adherence with documentation expectations as outlined by the collaborative practice agreement.

\section{METHODS}

The DWG conducted a retrospective audit of chart documentation by pharmacists in the fourth quarter of 2017. Our institution did not require approval from the ethics review board for this quality improvement initiative. A drug use evaluation report was generated to identify patients who received at least 1 dose of vancomycin, gentamicin, or tobramycin in the month before and including the audit date. A convenience sample of the patients on this list who remained as inpatients at either the Pasqua Hospital or the Regina General Hospital on the date of the audit was selected, based on ease of evaluator access to patient charts.

The audit consisted of 2 distinct components. In the first component, 3 members of the DWG reviewed the charts to determine the presence or absence of any progress notes, as outlined in the documentation requirements of the pharmacist procedures supporting the collaborative agreement. Notes were stratified by type, either as notes for empiric therapy or as follow-up notes after measurement of serum levels. For the second component, a random subset of these 
notes was selected for further analysis. Seven DWG members (including L.R. and C.G.) reviewed the notes to determine the presence of each specific element outlined in the procedure. The results of both components of the audit were reviewed by the DWG and next steps established.

An educational session was conducted to present results of the first audit, reinforce procedural requirements, and engage pharmacy staff in evaluation of various sample notes, applying the same assessments as were used by DWG members.

A second audit was completed in the fourth quarter of 2018, using the same methods as the first, to assess adherence to documentation requirements. A data collection tool was utilized for the second audit to build greater consistency among assessors, although the factors assessed remained the same.

\section{RESULTS}

In the first audit, a total of 51 charts were evaluated, with identification of 162 instances requiring documentation, as per the procedure. A note was present in 137 of these instances $(84.6 \%$ adherence rate). In the second audit, 88 charts were evaluated. Notes were present in 253 of the 295 instances where notes were expected (85.8\% adherence rate). Table 1 depicts adherence rates stratified by note type. In both audits, the vast majority of patients whose charts were reviewed (98\%) had received vancomycin.

Table 2 and Table 3 depict the breakdown of adherence to documentation requirements for specific elements in the empiric therapy notes and the notes prepared after measurement of serum levels, respectively. The elements most commonly omitted from empiric therapy notes included specification of a monitoring plan for evaluation of serum levels and serum creatinine. Notes documented after measurement of serum level frequently lacked assessment of the appropriateness of the measured serum level, interpretation of serum level results, and specification of duration of therapy.

\section{DISCUSSION}

Documentation is an integral component of the collaborative practice agreement model, intended to increase the clarity of the decision-making rationale among health care team members, as specified by legal and professional requirements. ${ }^{1-4}$ According to the SHA - Regina area collaborative practice agreements for management of vancomycin and aminoglycoside therapy, it is expected that all interventions by pharmacists will be documented within the medical record. In this study, the DWG found that documentation was present in $84.6 \%$ and $85.8 \%$ of expected instances in the first and second audits, respectively. Although the documentation of empiric therapy notes tended to be higher than for follow-up notes, the absence of any documentation on initiation of therapy in $5 \%-10 \%$ of evaluated patient charts is concerning. In addition, 9 of the audited charts had no notes indicating a pharmacist's involvement in collaborative prescribing. These omissions may have resulted from a lack of easy access to the paper-based patient chart, initiation of therapy during hours of limited pharmacist availability, or pharmacists' perceptions that notes are not reviewed extensively by other health care providers, making the inclusion of a note a lesser priority. Further study of the factors leading to these omissions, with identification of supportive actions to achieve complete documentation in all cases, is required.

All note components were weighted equally within the audits. Omissions of date, time, pharmacist identification outside of signature, and contact information all contributed to non-adherence. Although all components are important, the DWG members felt there was greatest potential impact on patient care when documentation related to assessment of serum levels, interpretation of results, action plan, and rationale for clinical decision-making was absent. These omissions remain consistent with those previously reported by Tangedal and others, ${ }^{8}$ although documentation was not the primary focus of their study. The most common omissions after measurement of serum levels occurred when dose adjustments made daily in the critical care population were documented solely in the physician's orders section of the chart, with no corresponding progress note documentation to provide the rationale or details of level assessment. The Steering Committee confirmed that this practice did not meet the intent of the collaborative practice agreement. This was re-emphasized during the educational session. In the second audit, documentation of serum level assessments increased from $39 \%$ to $66 \%$, interpretation of results from $55 \%$ to $82 \%$, and action plan and dose adjustments from $68 \%$ to $97 \%$ (Table 3 ).

TABLE 1. Frequency of Note Presence in Patient Chart Progress Notes

\begin{tabular}{lrrrr} 
& \multicolumn{3}{c}{$\begin{array}{c}\text { Audit Number; } \\
\text { Note Type }\end{array}$} & \multicolumn{2}{c}{ No. of Notes Present/Expected (\%) } \\
\cline { 2 - 5 } & \multicolumn{2}{c}{ First Audit } & \multicolumn{2}{c}{ Second Audit } \\
\hline Empiric therapy & $65 / 69$ & $(94.2)$ & $86 / 96$ & $(89.6)$ \\
After measurement of serum level & $72 / 93$ & $(77.4)$ & $167 / 199$ & $(83.9)$ \\
Total & $137 / 162$ & $(84.6)$ & $253 / 295$ & $(85.8)$ \\
\hline
\end{tabular}


TABLE 2. Inclusion of Required Elements of Empiric Therapy Notes, as Specified in Vancomycin-Aminoglycoside Collaborative Prescribing Procedures ${ }^{\mathrm{a}}$

Audit Number; No. (\%) of Notes

\begin{tabular}{lllll} 
Note Element & $\begin{array}{c}\text { First Audit } \\
(\boldsymbol{n}=\mathbf{2 6})\end{array}$ & $\begin{array}{c}\text { Second Audit } \\
(\boldsymbol{n}=\mathbf{8 0})\end{array}$ \\
\hline Date and time of note & 18 & $(69)$ & 54 & $(67)$ \\
\hline Indication & 19 & $(73)$ & 74 & $(92)$ \\
\hline Desired target trough & 19 & $(73)$ & 68 & $(85)$ \\
\hline Nephrotoxic risk factors & 24 & $(92)$ & 62 & $(77)$ \\
\hline Frequency of serum creatinine monitoring & 13 & $(50)$ & 52 & $(65)$ \\
\hline $\begin{array}{l}\text { Serum creatinine (date of result and creatinine } \\
\text { clearance calculation) }\end{array}$ & 17 & $(65)^{b}$ & 43 & $(54)$ \\
\hline Interval based on estimated creatinine clearance & & & 62 & $(77)$ \\
\hline Loading dose, if required & 22 & $(85)$ & 70 & $(87)$ \\
\hline Dose, including weight & 22 & $(85)$ & 59 & $(74)$ \\
\hline When serum levels expected, if at all & 17 & $(65)$ & 70 & $(87)$ \\
\hline Name of note writer (printed and signed) & 12 & $(46)^{c}$ & 56 & $(70)$ \\
\hline Writer's contact information & & & $51 \quad(64)$
\end{tabular}

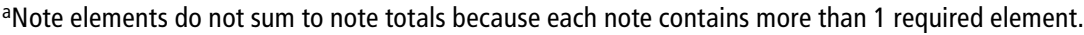
${ }^{b}$ In the first audit, data for date of serum creatinine result and clearance calculation were combined with dosing interval based on the estimate creatinine clearance.

In the first audit, data for name and signature of note writer were combined with data for contact information.

TABLE 3. Inclusion of Required Note Elements after Assessment of Serum Level, as Specified in Vancomycin-Aminoglycoside Collaborative Prescribing Procedures ${ }^{a}$

Audit Number; No. (\%) of Notes

\begin{tabular}{|c|c|c|c|}
\hline Note Element & $\begin{array}{l}\text { First Audit } \\
\quad(n=38)\end{array}$ & $\begin{array}{r}\text { Seco } \\
(n\end{array}$ & $\begin{array}{l}\text { Audit } \\
19)\end{array}$ \\
\hline Date and time of note & $31 \quad(82)$ & 99 & (83) \\
\hline Date and time of serum level & $22 \quad(58)$ & 63 & $(53)$ \\
\hline Results reported in $\mathrm{mg} / \mathrm{L}$ & $26 \quad(68)$ & 116 & (97) \\
\hline Any discussion with prescriber & NA & \multicolumn{2}{|c|}{ NA } \\
\hline $\begin{array}{l}\text { Assessment of serum level (e.g., "sample drawn } \\
\text { appropriately at steady state") }\end{array}$ & $15 \quad(39)$ & 79 & $(66)$ \\
\hline Interpretation of result & $21 \quad(55)$ & 97 & $(82)$ \\
\hline Action plan, including dose adjustment if required & $26 \quad(68)$ & 115 & (97) \\
\hline When next level due & $21 \quad(55)$ & 94 & (79) \\
\hline Duration of treatment & $4 \quad(11)$ & 43 & (36) \\
\hline Name of note writer (printed and signed) & $19(50)^{b}$ & 36 & $(30)$ \\
\hline Writer's contact information & & 40 & (34) \\
\hline
\end{tabular}

$\mathrm{NA}=$ not available (expectation that discussions with prescribers would be documented, as per procedure, but unable to confirm by means of methodology used in this study).

a Note elements do not sum to note totals, because each note contains more than 1 required element. bIn the first audit, data for name and signature of note writer were combined with data for contact information. 
Professional guidelines (from the Canadian Society of Hospital Pharmacists ${ }^{2}$ and the American Society of Health-System Pharmacists ${ }^{1}$ ) have not established defined criteria for adequate documentation, although a requirement for complete documentation can be inferred. A relative paucity of data exists examining pharmacists' prescribing within a collaborative practice framework. A study in Calgary that was designed to evaluate independent pharmacist prescribing practices in a population of acute care inpatients found, as a secondary outcome, that documentation occurred in $58 \%$ of a random sample of 50 patient charts audited. ${ }^{10}$ Adam and others ${ }^{11}$ assessed the rates of documentation by clinical pharmacists throughout a patient's hospital stay, utilizing chart assessment processes similar to our audits. In their study, most patients received "minimal documentation" (72.3\%) defined as at least 1 intervention described in writing, rather than "extensive documentation" (10.4\%), defined as 2 or 3 notes, primarily at points of transitions in care. ${ }^{11}$ These studies do not directly relate to the expectations required within collaborative agreements, but they reinforce recognition of the importance of complete documentation by pharmacists, as well as recognition of gaps between expectations and performance.

Numerous factors may have affected our results. The assessors differed between the 2 audits, potentially increasing the risk for observational bias. In an attempt to build greater consistency, a more rigorous data collection tool was used for the second audit, which might have affected the assessments. Notes were selected for assessment at random and did not encompass all pharmacists included within the collaborative agreement. The complement of pharmacists changed during the auditing timeframe, with $26 \%(14 / 54)$ of our pharmacist team having less than 2 years of experience at the time of the second audit. The impact of staff change is not known, as the audit was not set up to compare results between pharmacists with more and less experience. Recent training may have been beneficial in ensuring awareness of requirements, although the opportunity for application of learning after receipt of training may have varied. Educational initiatives to improve documentation have been supported in the literature, with emphasis on the need for ongoing and repetitive reinforcement. ${ }^{12}$ Although our collaborative prescribing agreement procedures were guided by a multidisciplinary steering committee, collaborative stakeholders were not surveyed directly to determine their perceptions about the adequacy of documentation to meet communication needs. Finally, given that pharmacists' documentation is strictly paper-based in the SHA - Regina area, these results may not be directly applicable to practices with access to electronic documentation.

Transitioning to electronic documentation by pharmacists in a patient's electronic chart, with templated requirements built into the software framework, may help to address the barriers to documentation that we identified, including lack of time, poor access to paper-based charts in a timely manner, and uncertainty as to what to document. ${ }^{9,11}$ Our organization is moving toward this technology in the future. As future collaborative prescribing roles are developed, agreements should clearly specify expectations and requirements for documentation. Engaging with stakeholders proactively during creation of these agreements may assist in ensuring that documentation meets requirements for all parties. Continuous quality improvement initiatives are integral to ensure that documentation expectations are met.

\section{CONCLUSION}

Although adherence to documentation requirements was high $(>84 \%)$, further investigation is needed to elucidate mechanisms for enhancing documented communication within collaborative prescribing situations. An educational intervention presented to staff after the first audit, to highlight documentation requirements, resulted in improved adherence in the second audit, with inclusion of components essential to patient care.

Utilization of electronic mechanisms for documentation and software supports to force documentation functions may be beneficial. Within the certification process, expectations for clear and complete documentation must be emphasized. Regular audits and education with all pharmacists should continue.

\section{References}

1. American Society of Health-System Pharmacists. ASHP guidelines on documenting pharmaceutical care in patient medical records. Am J Health Syst Pharm. 2003;60(7):705-7.

2. Documentation of pharmacists' activities in the health record: guidelines. Canadian Society of Hospital Pharmacists; 2013.

3. Model standards of practice for Canadian pharmacists. National Association of Pharmacy Regulatory Authorities; 2009.

4. The regulatory bylaws of the Saskatchewan College of Pharmacy Professionals. Saskatchewan College of Pharmacy Professionals; 2019.

5. Collaborative practice (prescribing) agreement template. In: Reference manual. Saskatchewan College of Pharmacy Professionals; 2017.

6. Quality assurance framework for enhanced authority for the pharmacist to prescribe drugs in collaborative practice environments. Saskatchewan College of Pharmacists; 2011.

7. Baranski B, Bolt J, Albers L, Siddiqui R, Bell A, Semchuk W. Development of a documentation rubric and assessment of pharmacists' competency for documentation in the patient health record. Can J Hosp Pharm. 2017;70(6):423-9.

8. Tangedal K, Bolt J, Len S, Bell A. Baseline competency assessment of pharmacists prescribing and managing vancomycin therapy in the Regina Qu'Appelle Health Region. Can J Hosp Pharm. 2017;70(5):335-42.

9. Zimmer S, Gray C, Roy C, Semchuk WM. Departmental initiative to improve documentation in the medical record by acute care pharmacists. Can J Hosp Pharm. 2019;72(2):151-4.

10. Saunders S, Dersch-Mills D, Mysak T, Romonko-Slack L, Chernick A, Lazarenko G, et al. CAPABLE: Calgary zone usage of additional prescribing authorization by pharmacists in an inpatient setting: review of the prescribing landscape and environment. Res Social Adm Pharm. 2020;16(3):342-98. 
11. Adam JP, Trudeau C, Pelchat-White C, Deschamps ML, Labrosse P, Langevin MC, et al. Documentation in the patient's medical record by clinical pharmacists in a Canadian university teaching hospital. Can J Hosp Pharm. 2019;72(3):194-201.

12. Rector KB, Veverka A, Krick Evans S. Improving pharmacist documentation of clinical interventions through focused education. Am J Health Syst Pharm. 2014;71(15):1303-10.
Brandon Kennedy, BSc, BSP, is with the Department of Pharmacy Services, Saskatchewan Health Authority - Regina, Regina, Saskatchewan.

Carolyn Gray, BSP, ACPR, is with the Department of Pharmacy Services, Saskatchewan Health Authority - Regina, Regina, Saskatchewan.

Lisa Ruda, BSP, ACPR, is with the Department of Pharmacy Services, Saskatchewan Health Authority - Regina, Regina, Saskatchewan.

William Semchuk, MSc, PharmD, FCSHP, is with the Department of Pharmacy Services, Saskatchewan Health Authority - Regina, Regina, Saskatchewan.

Competing interests: For activities unrelated to the study reported here William Semchuk has received personal fees from Bayer, Pfizer, BMS, AstraZeneca, Servier, and Sanofi. No other competing interests were declared.

Address correspondence to:

Carolyn Gray

Department of Pharmacy Services

Saskatchewan Health Authority - Regina

1440 14th Avenue

Regina SK S4P 0W5

email: Carolyn.gray@saskhealthauthority.ca

Funding: None received.

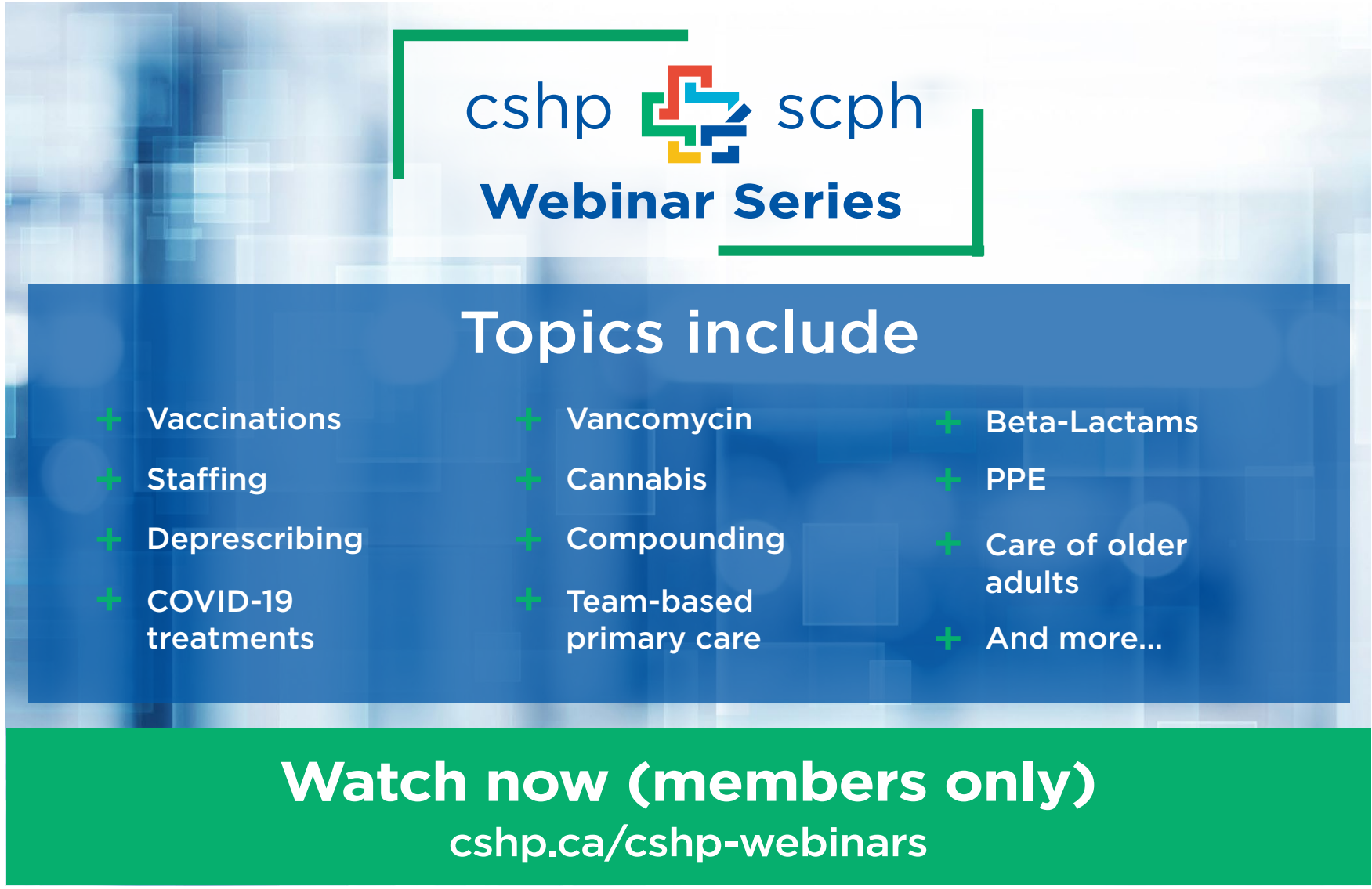

